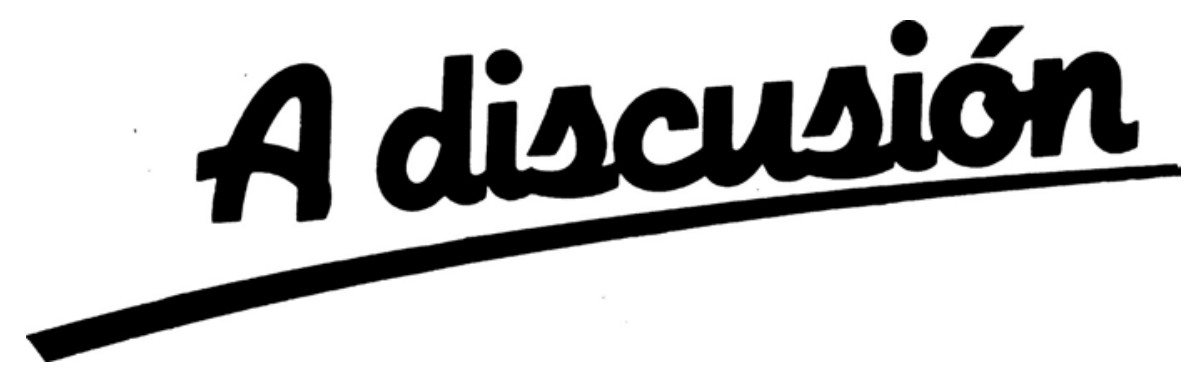

\title{
CONDORCET CHOICE FUNCTIONS AND MAXIMAL ELEMENTS*
}

\section{Begoña Subiza and Josep E. Peris**}

WP-AD 2003-40

Correspondence to: B. Subiza, Universidad de Alicante, Departamento de Fundamentos del Análisis Económico, Campus San Vicente del Raspeig, Ap. Correos, 99. 03080 Alicante (Spain). E-mail: subiza@merlin.fae.ua.es.

Editor: Instituto Valenciano de Investigaciones Económicas, S.A.

Primera Edición Diciembre 2003

Depósito Legal: V-5479-2003

IVIE working papers offer in advance the results of economic research under way in order to encourage a discussion process before sending them to scientific journals for their final publication.

\footnotetext{
* Thanks are due to José Alcalde, J.V. Llinares and M.C. Sánchez, for helpful comments. The comments and suggestions of a referee's report have improved the work; we are most grateful to her/him. Financial support from the Spanish DGICYT, under project BEC2001-0781, Fundación Seneca (PI74/00826/FS/01) and from the IVIE is acknowledged.

** B. Subiza and J.E. Peris: Departamento de Fundamentos de Análisis Económico, Universidad de Alicante, Campus San Vicente del Raspeig, 03071 Alicante, Spain, subiza@merlin.fae.ua.es, peris@merlin.fae.ua.es.
} 
CONDORGET GHOIGE FUNGTIONS AND MAXIMAL ELEMENTS

\author{
Begoña Subiza and Josep E. Peris
}

\begin{abstract}
Choice functions on tournaments always select the maximal element (Condorcet winner), provided they exist, but this property does not hold in the more general case of weak tournaments. In this paper we analyze the relationship between the usual choice functions and the set of maximal elements in weak tournaments. We introduce choice functions selecting maximal elements, whenever they exist. Moreover, we compare these choice functions with those that already exist in the literature.
\end{abstract}

Key words: choice functions, tournaments, maximal elements.

JEL classification number: D70 


\section{Introduction}

In the literature on preferences, there are a great number of papers devoted to the analysis of the existence of maximal elements, as they are considered "the best ones". In order to ensure the existence of maximal elements in all feasible subsets of $X$, the acyclicity of the binary relation $P$ is a necessary condition; but there are many contexts in which requiring acyclicity of the binary relation a priori is too rigid a restriction. This is the case of realistic social decision mechanisms as, for instance, majority voting in which cycles may appear. Moreover, requiring acyclicity, together with some other axioms, in social decision functions gives rise to impossibility results. As pointed out by Schwartz (1986), "... the impossibility theorems show acyclicity to be unreasonable as a general assumption about collective preference -although not necessarily unreasonable when restricted to special situations. It seems unreasonable as a general assumption about individual preferences as well".

This fact has inspired many papers on the problem of choosing the best elements when binary relations are not necessarily acyclic, as in the case of tournaments (asymmetric and complete binary relations), or weak tournaments where the binary relation is merely complete (this generalization of the notion of tournaments is interesting because it allows indifferences or ties). In these contexts, because of the non-existence, in general, of maximal elements, it is not quite clear what the definition of "the best elements" is, and several solution concepts (choice functions), from both the positive and the axiomatic points of view, have been introduced. 
In the case of tournaments, the relation between the selected elements for any well-defined choice function and the maximal elements is always clear: the existence of maximal elements cannot be ensured, but if there is a maximal element, it will be the one that beats all other alternatives (Condorcet winner) and the choice function selects precisely such an element. In tournaments, therefore, the choice set and the set of maximals (if it is nonempty) coincide.

In the case of weak tournaments, however, the relationship between maximal elements and choice functions is not so clear and, as we will show, the choice functions defined in the literature for this case do not always coincide with the set of maximal elements: some of them select a larger set (with regard to the inclusion relation), while others may select elements of which none of them is in fact maximal.

We are interested in defining a choice function, in the framework of weak tournaments, such that it chooses maximal elements, whenever they exist, and then generalizes what happens in the case of tournaments.

\section{Weak Tournaments and Maximal Elements}

Let us consider the following definitions and notation.
A tournament $T=(X, U)$ consists of a non-empty finite set of alternatives $X$ and a binary relation $U$ defined on $X$ which is complete $[\forall x, y \in X, x \neq y \Rightarrow x U y$ or $y U x]$ and asymmetric $[x U y \Rightarrow \operatorname{not}(y U x)] . \quad$ A weak tournament $W=$ 
$(X, R)$ is a generalization of the previous concept which only requires strong completeness of the binary relation $R \quad[\forall x, y \in X, \Rightarrow x R y$ or $y R x]$. We denote the family of tournaments by $\mathcal{T}$, while $\mathcal{W}$ represents the family of weak tournaments.

A choice function $F$ is a map that assigns a non-empty choice set (the selected alternatives) in $X$, to each tournament or weak tournament. Given two choice functions $F, G, F \neq G$, it is said that $F$ is contained in $G$ (or that $F$ is more discriminating than $G$ ), denoted as $F \subset G$, if for all weak tournaments $W=(X, R)$, $F(W) \subseteq G(W)$.

Given a weak tournament $W=(X, R)$, for each subset $A$ of $X$, the maximal set is denoted by $M(A, R)=\{x \in A \mid x R y, \forall$ $y \in A, \quad y \neq x\}$. Under the standard interpretation, $M(A, R)$ can be interpreted as the best elements in $A$, for the binary relation $R$. From $R$, its asymmetric part $P$ is defined as follows: $x P y$ if and only if $[x R y$ and $\operatorname{not}(y R x)]$. The symmetric part of $R$, (in which two elements tie, or are indifferent), is defined as follows: $x I y$ if and only if $[x R y$ and $y R x]$. The transitive closure of a binary relation $R$ on $X$, denoted by $R^{\infty}$, is defined as follows: $x R^{\infty} y$ if and only if there are $x_{1}, x_{2}, \ldots, x_{n} \in X$ such that $x=x_{1} R x_{2} R \ldots$ $R x_{n}=y$. The transitive closure of the relation $P$ on $X$ is defined analogously: $x P^{\infty} y$ if and only if there are $x_{1}, x_{2}, \ldots, x_{n} \in X$ such 
that $x=x_{1} P x_{2} P \ldots P x_{n}=y$. Note that, in general, $P^{\infty}$ does not coincide with the asymmetric part of $R^{\infty}$.

Given a weak tournament $W=(X, R)$ and $A \subseteq X,\left.W\right|_{A}$ denotes the weak tournament $\left(A,\left.R\right|_{A}\right)$ where $\left.R\right|_{A}$ is the restriction of $R$ on the subset $A$. We now use the following function $g_{W}$ : $X \times X \rightarrow\{-1,0,1\}$ to completely represent a weak tournament $W=(X, R)$ :

$$
g_{W}(x, y)=\left\{\begin{array}{rrr}
1 & \text { if } & x P y \\
-1 & \text { if } & y P x \\
0 & & \text { otherwise }
\end{array}\right.
$$

or, in a matrix form, if $X=\left\{x_{1}, \ldots, x_{r}\right\}$,

$$
G_{W}=\left(\left(G_{W}\right)_{i j}\right)_{r \times r}, \quad\left(G_{W}\right)_{i j}=g_{W}\left(x_{i}, x_{j}\right) .
$$

Remark 1 A comparison function (Dutta and Laslier, 1999) is a generalization of weak-tournaments in which the skew-symmetric function $g_{W}$ admit the possibility of taking into account the intensity of the preference of $x$ over $y$. For comparison functions $g_{W}$ with values in $\{-1,0,1\}$, we obtain the particular case of weak-tournaments.

\subsection{Choice functions for weak tournaments}

The choice functions defined for tournaments satisfy the following property (Condorcet choice functions):

For all $T=(X, U) \in \mathcal{T}$,

$$
F(T)=\{x\} \text { whenever } x U y, \forall y \in X, x \neq y
$$


or, in other words,

$$
F(T)=M(X, U) \text { whenever the maximal set is non-empty. }
$$

As we have already mentioned, this is not the case for choice functions defined for weak tournaments. In order to show this, we analyze Condorcet choice functions that have been generalized to the context of weak tournaments:

The top cycle, denoted as $T C$, is the first reference for a solution to a tournament $T=(X, U) \in \mathcal{T}$, and it is defined by the elements $x \in X$ such that $x U^{\infty} y$ for all $y \in X, y \neq x$. Its extension for weak tournaments $T C(W)$, known as the GOCHA set (Schwartz, 1972), is defined as the maximal elements in $X$ of the transitive closure $R^{\infty}$.

The uncovered set (Fishburn, 1977; Miller, 1980) of a tournament $T=(X, U) \in \mathcal{T}$, is the subset $U C(T)$ of $X$, whose members are maximal elements of the covering relation defined by: $x$ covers $y$ if and only if $[x U y$ and $(y U z$ implies $x U z)]$. Dutta (1988), defines the minimal covering set on $\mathcal{T}$, denoted as $M C(T)$, based on the idea of covering sets. A covering set of a tournament $T=(X, U) \in \mathcal{T}$ is a non-empty subset $A$ of $X$ such that $U C\left(\left.T\right|_{A}\right)=A$, and for all $x \in X-A, x \notin U C\left(\left.T\right|_{A \cup\{x\}}\right)$. The minimal covering is a covering set $B$ such that no proper subset of $B$ is a covering set. Dutta (1988) proves that for any tournament $T$, such a subset always exists and is unique. These two choice functions have been extended for weak tournaments 
(Peris and Subiza, 1999) in such a way that they satisfy the same axioms as in tournaments.

Another important solution concept for tournaments that has been generalized to weak tournaments is the Bipartisan set (Laffond, Laslier and Le Breton, 1993). For a tournament $T=(X, U) \in \mathcal{T}$, consider the following two-player symmetric zero-sum game: both players have $X$ as their set of pure strategies and the payoff of the first player is given by the function $g_{T}$. This game has a unique equilibrium in mixed strategies and the authors define the choice function $B P(T)$ (the Bipartisan set) as the support of such an equilibrium. The extension of the Bipartisan set to comparison functions (Dutta and Laslier, 1999), called the Essential set, $E S(W)$, is defined as the union of the supports of all the Nash equilibria of the two-player symmetric zero-sum game, in which both players have $X$ as their set of pure strategies and the payoff of the first player is given by the function $g_{W}$

Laffond, Laslier and Le Breton (1995) provide a set-theoretical comparison of the previous choice functions in the context of tournaments. Specifically, they obtain that in $\mathcal{T}$

$$
B P \subset M C \subset U C \subset T C
$$

For weak tournaments the set-comparisons are the same (see Peris and Subiza, 1999; Dutta and Laslier, 1999), 


$$
E S \subset M C \subset U C \subset T C .
$$

When applied to tournaments, all the above-mentioned solutions are Condorcet choice functions, so that if there is a maximal element, all solutions agree in their choice of the Condorcet winner. Let us show that this is not necessarily true when it is applied to weak tournaments.

Example 1 Let $W=(X, R)$ the weak tournament where $X=\{a, b, c\}$ and $a I b, a P c, c P b . \quad M(X, R)=\{a\}$, but $E S(W) \supseteq\{a, b\}$ because $\left(\left(\frac{1}{2}, \frac{1}{2}, 0\right),\left(\frac{1}{2}, \frac{1}{2}, 0\right)\right)$ is an equilibrium of the zero-sum game defined by this weak tournament.

In the following proposition we prove that the maximal elements, when they exist, are included in the essential set, and therefore, in the minimal covering, the uncovered set and the top cycle.

Proposition 1 Given a weak tournament $W=(X, R)$,

$$
M(X, R) \subset E S(W) .
$$

Proof. Consider the zero-sum game defined by the payoff function $g_{W}$ associated to the weak tournament $W=(X, R)$. Then if $x \in M(X, R)$, $g_{W}(x, y) \in\{0,1\}$ for all $y \in X$; since $g_{W}(x, x)=0,(x, x)$ is a saddle-point and $x \in E S(W)$. Moreover, Example 1 shows that this inclusion may be proper. 


\subsection{Generalized Condorcet condition}

To expect acyclicity of the binary relation (that is, to insist on the existence of maximal elements in every subset of the feasible set) is not reasonable when considered as a global assumption. Nevertheless, it is possible that maximal elements exist in some subsets (for instance, in subsets with just two elements). Whenever maximal elements exist, a desirable property of a choice function is that it selects these maximal elements exclusively. Let us call the choice functions that satisfy this property general Condorcet choice functions:

Definition 1 A choice function $F$ defined in the family of weak tournaments is said to be a $\boldsymbol{g}$-Condorcet choice function if for each weak tournament $W=(X, R) \in \mathcal{W}$, such that $M(X, R) \neq \emptyset$ then $F(W)=M(X, R)$.

If we have a tournament, this notion corresponds to that of a Condorcet choice function. Moreover, if the binary relation is acyclic, a g-Condorcet choice function coincides with the concept of a rational choice function (for this reason, we will call rational the choice functions satisfying the gCondorcet property). As shown in Example 1, the afore-mentioned solution concepts for weak tournaments do not satisfy this condition, since they may all choose "bad" candidates (elements which are not in $M(X, R)$ ). This fact is somewhat related with the notions of Type1-Type2 choice functions (Dutta and Laslier, 1999). Most of choice functions defined in the literature about weak-tournaments are Type 1 choice functions, which requires that in the 
situation shown in Example 1, the whole set $X=\{a, b, c\}$ must be chosen. In this paper we are interested in Type 2 (not Type 1) choice functions.

\section{3 g-Condorcet Choice Functions for Weak Tournaments}

Before introducing new choice functions satisfying the g-Condorcet property, we need some notation. For any weak tournament $W=(X, R)$, consider the following equivalence relation defined on $X: x \approx y$ if and only if $x=y$ or $\left[x P^{\infty} y\right.$ and $\left.y P^{\infty} x\right]$. We denote the quotient set by $\mathbb{X}$, and by $\operatorname{cl}(x ; W)$ the class in $\mathbb{X}$ containing the element $x \in X$ [if there is no risk of confusion, we merely write $\operatorname{cl}(x)]$. We now define the quotient relation $\mathbb{P}$ on $\mathbb{X}$ as follows:

for all $\operatorname{cl}(x), c l(y) \in \mathbb{X}$,

$$
\begin{aligned}
& \operatorname{cl}(x) \mathbb{P} c l(y) \text { if and only if } \\
& \text { a) } \operatorname{cl}(x) \neq c l(y) \text {, and } \\
& \text { b) there exist } a \in \operatorname{cl}(x), b \in \operatorname{cl}(y) \text { such that } a P^{\infty} b \text {. }
\end{aligned}
$$

And, from $\mathbb{P}$ we define

$$
\operatorname{cl}(x) \mathbb{R} c l(y) \text { if and only if } \operatorname{not}[\operatorname{cl}(y) \mathbb{P} c l(x)] .
$$

It is easy to prove that $\mathbb{P}$ is an asymmetric and transitive binary relation, so that $M(\mathbb{X}, \mathbb{R})$ is a non-empty set (see Peris and Subiza, 1994). We know that, given a preference relation $R$, maximal elements exist if and only if the quotient relation has maximal classes with cardinality 1. Therefore, if we wish to define a choice function that satisfies the g-Condorcet condition, 
we must consider only the classes with cardinality 1 , whenever they exist. This fact induces us to select the maximal classes with the lowest possible cardinalities: we denote the classes in $M(\mathbb{X}, \mathbb{R})$ with minimal cardinality by $m c M(W)$,

$$
m c M(W)=\{c l(x) \in M(\mathbb{X}, \mathbb{R}) \mid \#(c l(x)) \leq \#(c l(y)), \forall \operatorname{cl}(y) \in M(\mathbb{X}, \mathbb{R})\}
$$

where

\#(·) denotes the cardinality of a finite set.

Definition 2 The Rational Top Cycle choice function assigns, to each weak tournament $W=(X, R)$, the set

$$
T C^{*}(W)=\bigcup_{c l(x) \in m c M(W)} c l(x) .
$$

In the following theorem, we prove that the Rational Top Cycle is a gCondorcet choice function that generalizes the top cycle to the case of weak tournaments.

\section{Theorem 1}

a) The Rational Top Cycle is a g-Condorcet choice function.

b) For all tournaments $T=(X, U) \in \mathcal{T}, T C^{*}(T)=T C(T)$.

c) $T C^{*} \subset T C$.

\section{Proof.}

a) For each weak tournament $W=(X, R)$ such that $M(X, R) \neq \emptyset$, each maximal element constitutes, by itself, a maximal class in $M(\mathbb{X}, \mathbb{R})$, although other maximal classes may exist in the quotient relation. The union of the classes with minimal cardinality coincides with the maximal set. 
b) If $T=(X, U) \in \mathcal{T}$, there is just one maximal class on $M(\mathbb{X}, \mathbb{R})$ which is precisely the Top Cycle of this tournament, $T C(T)$.

c) The inclusion is obvious from the fact that the top cycle may be written as:

$$
T C(W)=\bigcup_{c l(x) \in M(\mathbb{X}, \mathbb{R})} c l(x)
$$

and Example 1 shows that $T C^{*}(W)$ may be strictly included in $T C(W)$,

$$
T C^{*}(W)=\{a\}, \quad T C(W)=\{a, b, c\} .
$$

Definition 3 The Rational Essential choice function assigns, to each weak tournament $W=(X, R)$, the set

$$
E S^{*}(W)=\bigcup_{c l(x) \in m c M(W)} E S\left(\left.W\right|_{c l(x)}\right) .
$$

\section{Theorem 2}

a) The Rational Essential is a g-Condorcet choice function.

b) For all tournament $T=(X, U), E S^{*}(T)=E S(T)$.

c) $E S^{*} \subset E S$.

\section{Proof.}

a) Analogous to part a) in the previous Theorem.

b) If $T=(X, U) \in \mathcal{T}$, there is just one maximal class on $M(\mathbb{X}, \mathbb{R})$ which is precisely the Top Cycle of this tournament, $T C(T)$. Therefore,

$$
E S^{*}(T)=E S\left(\left.T\right|_{T C(T)}\right)=E S(T) .
$$

c) Let $x \in E S^{*}(W)$; then $x \in E S\left(\left.W\right|_{c l(x)}\right)$, where $c l(x) \in m c M(W)$. Let $g_{W c l(x)}$ be the payoff function associated to the weak-tournament $\left(\left.W\right|_{c l(x)}\right)$. Then, $x \in \operatorname{supp}\left(p^{*}\right)$, where $\left(p^{*}, p^{*}\right)$ is a Nash equilibrium of the zero-sum game 
with payoff function $g_{W c l(x)}$. Let us consider the following strategy of the game defined by $g_{W}$, the payoff function associated to the weak-tournament $W$,

$$
\begin{array}{ll}
p^{\prime}(y)=p^{*}(y) & \text { for all } y \in \operatorname{cl}(x), \\
p^{\prime}(y)=0 & \text { otherwise. }
\end{array}
$$

Let us see that $\left(p^{\prime}, p^{\prime}\right)$ is a Nash equilibrium of the game $g_{W}$. To do so, it is sufficient (see, for instance, Owen, 1982) to prove that

$$
\begin{array}{ll}
\text { (1) } \forall z \in \operatorname{supp}\left(p^{\prime}\right) & \sum_{z P y} p^{\prime}(y)=\sum_{y P z} p^{\prime}(y) \text {, and that } \\
\text { (2) } \forall z \notin \operatorname{supp}\left(p^{\prime}\right) & \sum_{z P y} p^{\prime}(y) \leq \sum_{y P z} p^{\prime}(y) .
\end{array}
$$

Condition (1) is fulfilled, since $\operatorname{supp}\left(p^{\prime}\right)=\operatorname{supp}\left(p^{*}\right)$. In order to verify (2), let us suppose first that $z \notin \operatorname{supp}\left(p^{\prime}\right)$, and $z \in \operatorname{cl}(x)$. Then,

$$
\sum_{z P y} p^{\prime}(y)=\sum_{z P y} p^{*}(y) \leq \sum_{y P z} p^{*}(y)=\sum_{y P z} p^{\prime}(y) .
$$

In this case, therefore, condition (2) is fulfilled. If, on the other hand, $z \notin \operatorname{cl}(x)$, then for all $y \in \operatorname{supp}\left(p^{\prime}\right), n o(z P y)$ and therefore

$$
\sum_{z P y} p^{\prime}(y)=0 \leq \sum_{y P z} p^{\prime}(y) .
$$

Finally, Example 1 shows that the inclusion may be proper.

Remark 2 In order to show that the inclusion may be proper even in the case where no maximal element exists, we use the following example: let $W=(X, R)$ be the weak tournament in which $X=\left\{x_{1}, \ldots, x_{7}\right\}$ and the function $g_{W}$ is given by the following matrix 


$$
\left(\begin{array}{rrrrrrr}
0 & 1 & -1 & 0 & 0 & 0 & 0 \\
-1 & 0 & 1 & 0 & 0 & 0 & 0 \\
1 & -1 & 0 & 0 & 0 & 0 & 0 \\
0 & 0 & 0 & 0 & 1 & 0 & -1 \\
0 & 0 & 0 & -1 & 0 & 1 & 0 \\
0 & 0 & 0 & 0 & -1 & 0 & 1 \\
0 & 0 & 0 & 1 & 0 & -1 & 0
\end{array}\right)
$$

Then, $E S^{*}(W)=\left\{x_{1}, x_{2}, x_{3}\right\}$ and $E S(W)=X$.

Just as we did with the Rational Essential choice function, we can also define the Rational Uncovered Set, or the Rational Minimal Covering as extensions of the corresponding choice functions on $\mathcal{T}$ that satisfy the gCondorcet property by setting:

$$
\begin{aligned}
& U C^{*}(W)=\bigcup_{c l(x) \in m c M(W)} U C\left(\left.W\right|_{c l(x)}\right) . \\
& M C^{*}(W)=\bigcup_{c l(x) \in m c M(W)} M C\left(\left.W\right|_{c l(x)}\right) .
\end{aligned}
$$

We have focused our attention on the Essential set, since it is more discriminating than the other choice functions.

\section{An Undominated Selection}

Apart from introducing the Essential Set (in the more general framework of comparison functions), Dutta and Laslier (1999) analyzed it axiomatically and found that, although it is contained in the Minimal Covering, it may still 
contain elements that are weakly dominated. To obtain an undominated selection of the Essential Set, they define a sequential elimination of dominated elements. But, in Dutta and Laslier's words, "...this process does produce a well-defined choice function. Unfortunately, the function does not have very good axiomatic properties [in particular, it does not satisfy Monotonicity]. Hence, it seems that the selection of weakly dominated alternatives is one price that has to be paid in the transition [from the case of tournaments] to the more complex world of comparison functions [which includes, as a particular case, weak tournaments]."

As we have proven in Theorem 2, the Rational Essential set is included in the Essential set, but as we will see in Example 2, it may still contain weak dominated alternatives. In this section, we define a selection of the Rational Essential set satisfying monotonicity, which no longer contains dominated alternatives. First of all, definitions of weak dominance and monotonicity are introduced, and then it is proven that a g-Condorcet choice function is not, in general, compatible with the elimination of weakly dominated alternatives.

Definition 4 Given a weak tournament $W=(X, R)$, it is said that alternative $y$ is weakly dominated by alternative $x$ in such a weak tournament if for all $z \in X, g_{W}(x, z) \geq g_{W}(y, z)$, with at least one strict inequality. The undominated set is defined as those alternatives in $X$ which are not weakly dominated by any other alternative,

$$
U D(W)=\{x \in X \mid \text { no element in } X \text { weakly dominates } x\} .
$$

Definition 5 A choice function $F$ defined in the family of weak tournaments 
satisfies Undominance if for all weak tournaments $W=(X, R), F(W) \subseteq$ $U D(W)$.

Undominance is a desirable property, but it is, in general, incompatible with the g-Condorcet property, since maximal elements may be weakly dominated. The following example shows this fact.

Example 2 Let $W=(X, R)$ be the weak tournament in which $X=$ $\left\{x_{1}, x_{2}, x_{3}\right\}$ and $x_{1} P x_{2}, x_{1} I x_{3}, x_{2} I x_{3}$. Then, any choice function which contains $M(X, R)=\left\{x_{1}, x_{3}\right\}$ does not satisfy Undominance, since $x_{1}$ weakly dominates $x_{3}$.

If we apply Undominance several times,

$$
U D^{1}(W)=U D(W) ; \quad U D^{2}(W)=U D(U D(W), R) ; \ldots
$$

we are eliminating, in a sequential way, alternatives that are weakly dominated in successive subsets of undominated elements of $X$. This sequence converges to a set $U D^{\infty}(W)$ that represents the elements that "survive" sequential elimination (Dutta and Laslier, 1999). Another desirable property of choice functions is that of Monotonicity, which can be stated in the following way:

Definition 6 A choice function $F$ defined in the family of weak tournaments satisfies Monotonicity if for all weak tournaments $W=(X, R), W^{\prime}=$ $\left(X, R^{\prime}\right)$ such that:

$$
\begin{aligned}
W= & W^{\prime} \text { except for some pair of elements }(x, y) \text { such that } \\
& g_{W^{\prime}}(x, y)>g_{W}(x, y)
\end{aligned}
$$


then, $x \in F(W) \Rightarrow x \in F\left(W^{\prime}\right)$.

Dutta and Laslier (1999) provide an example showing that the choice function defined by

$$
S(W)=E S\left(U D^{\infty}(W)\right)
$$

does not satisfy Monotonicity. We will use the same example to show the impossibility of finding a choice function that satisfies Monotonicity and sequential elimination of weakly dominated alternatives:

Example 3 (Dutta and Laslier, 1999) Let $X=\left\{a_{1}, a_{2}, a_{3}, b_{1}, b_{2}, b_{3}\right\}$ and the weak tournaments defined below

$$
\begin{aligned}
& g_{W} \equiv\left(\begin{array}{rrrrrr}
0 & 1 & 0 & 0 & 0 & 0 \\
-1 & 0 & 1 & 0 & 0 & 0 \\
0 & -1 & 0 & 0 & 0 & 0 \\
0 & 0 & 0 & 0 & 1 & 0 \\
0 & 0 & 0 & -1 & 0 & 1 \\
0 & 0 & 0 & 0 & -1 & 0
\end{array}\right) \\
& g_{W^{\prime}}=g_{W} \quad \text { except } \\
& g_{W^{\prime \prime}}=g_{W} \quad \text { except } \quad g_{W^{\prime}}\left(a_{1}, a_{3}\right)=1
\end{aligned}
$$

If we consider a non-empty valued choice function $S$ satisfying sequential Undominance, then:

$$
S(W) \subseteq\left\{a_{1}, b_{1}\right\} \quad S\left(W^{\prime}\right)=\left\{b_{1}\right\} \quad S\left(W^{\prime \prime}\right)=\left\{a_{1}\right\}
$$

which is incompatible with Monotonicity.

We now introduce a selection of the Rational Essential choice function by choosing, from the elements in $E S^{*}(W)$, those that are not weakly 
dominated.

Definition 7 To each weak tournament $W=(X, R)$, the UndominatedEssential choice function assigns the set

$U E S(W)=E S^{*}(W) \cap U D(W)$.

\section{Theorem 3}

a) $\emptyset \neq U E S \subset E S^{*}$.

b) For any weak tournament $W=(X, R)$ such that $M(X, R) \neq \emptyset$,

$$
U E S(W) \subseteq M(X, R) .
$$

c) The UES choice function satisfies Monotonicity.

d) The UES choice function satisfies Undominance.

\section{Proof.}

a) The fact that $U E S \subset E S^{*}$ follows directly from the definition. Therefore, we must only prove the non-emptiness of this choice function.

(1) Suppose that there is a maximal element; then it can only be dominated by another maximal element, so there is a maximal element which is not dominated and the choice function is non-empty, since it coincides with the maximal elements which are undominated.

(2) Suppose now that there are no maximal elements (in this case, the classes in $M(\mathbb{X}, \mathbb{R})$ will contain, at least, 3 elements). First note that an element in a maximal class can only be dominated by another element in that class. If, in the contrary, $\operatorname{cl}(x)$ is a maximal class such that there is $y \notin \operatorname{cl}(x)$ weak-dominating $x$, we know that $x P z$ for some $z \in \operatorname{cl}(x)$ and then 
domination implies $y P z$, that is, $\operatorname{cl}(y) \mathbb{P} c l(x)$, contradicting that $c l(x)$ is a maximal class.

We know that the Essential set, applied to the maximal classes of minimal cardinality, contains undominated elements in this class (see Dutta and Laslier, 1999), and then undominated in the whole set of alternatives.

b) Obvious from the definition of $U E S$ and from the fact that $E S^{*}$ is a g-Condorcet choice function.

c) Let $W=(X, R), W^{\prime}=\left(X, R^{\prime}\right)$ be two weak tournaments such that

$$
\begin{aligned}
& W=W^{\prime} \text { except for some pair of elements }(x, y) \text { such that } \\
& g_{W^{\prime}}(x, y)>g_{W}(x, y)
\end{aligned}
$$

and suppose $x \in U E S(W)$. Then, $\operatorname{cl}(x ; W) \in m c M(W)$. We have the following possibilities:

(1) $y \notin \operatorname{cl}(x ; W):$

then $\operatorname{cl}\left(x ; W^{\prime}\right)=\operatorname{cl}(x ; W)$, and $x \in E S^{*}\left(W^{\prime}\right)$. Moreover, it is obvious that if $x \in U D(W)$, then $x \in U D\left(W^{\prime}\right)$, so $x \in U E S\left(W^{\prime}\right)$.

(2) $y \in \operatorname{cl}(x ; W)$ : then, as $W=W^{\prime}$ except for $x$ and $y$, the other classes remain unchanged, and for the class $c l\left(x, W^{\prime}\right)$ we have two possibilities: $\operatorname{cl}\left(x ; W^{\prime}\right)=\operatorname{cl}(x, W)$ or $c l\left(x, W^{\prime}\right) \subset \operatorname{cl}(x, W)$.

(2a) $\operatorname{cl}\left(x ; W^{\prime}\right)=\operatorname{cl}(x ; W)$ :

The same argument as in case (1) applies, by virtue of the fact that the Essential choice function, which is applied to the maximal class, satisfies Monotonicity.

(2b) In this case, we can write

$$
c l(x ; W)=\operatorname{cl}\left(x ; W^{\prime}\right) \cup B
$$


As $x P^{\infty} b$ for all $b \in \operatorname{cl}(x ; W)$, then $x\left(P^{\prime}\right)^{\infty} b$, so $c l\left(x ; W^{\prime}\right)$ is a maximal class. If for some $b \in \operatorname{cl}(x ; W), b\left(P^{\prime}\right)^{\infty} x$, then $b \in \operatorname{cl}\left(x ; W^{\prime}\right)$. In other case, $b \in B$, so we obtain that for all $a \in \operatorname{cl}\left(x ; W^{\prime}\right)$ and $b \in B, a\left(P^{\prime}\right)^{\infty} b$. Then $x$ belongs to a maximal class with minimal cardinality (the cardinality of this class has decreased and the other classes are not modified). Let us show that $x \in E S\left(\left.W^{\prime}\right|_{c l\left(x, W^{\prime}\right)}\right)$. We know that $x \in E S\left(\left.W\right|_{c l(x, W)}\right)$ and, by Monotonicity, $x \in E S\left(\left.W^{\prime}\right|_{c l(x, W)}\right)$, so there is an equilibrium $(p, p)$ in the game defined by $\left(\left.W^{\prime}\right|_{c l(x, W)}\right)$ such that $p(x)>0$. Consider the following splitting of the equilibrium vector and the payoff matrix, where the first component corresponds to the alternatives in $\operatorname{cl}\left(x, W^{\prime}\right)$, and the second one to the ones in $B$,

where we know that:

$$
p=\left(p_{1}, p_{2}\right), \quad G_{W^{\prime} \mid c l(x ; W)}=\left(\begin{array}{ll}
G_{11} & G_{12} \\
G_{21} & G_{22}
\end{array}\right),
$$

$$
G_{12} \geqq 0, \quad G_{21}=-G_{12} \leqq 0 .
$$

It is now easy to prove that

$$
\bar{p}_{1}=\alpha^{-1} p_{1}, \text { where } \alpha=\sum_{z \in c l\left(x ; W^{\prime}\right)} p(z),
$$

is an equilibrium in the game with payoff matrix $G_{11}$, which implies $x \in$ $E S\left(\left.W^{\prime}\right|_{c l\left(x ; W^{\prime}\right)}\right)$. Therefore, $x \in U E S\left(W^{\prime}\right)$.

d) Follows directly from definition. 


\section{References}

1. Dutta, B. (1988). "Covering sets and a new Condorcet Choice Correspondence", Journal of Economic Theory 44, 63-80.

2. Dutta, B. and J.F. Laslier (1999). "Comparison Functions and Choice Correspondences", Social Choice and Welfare 16, 513-532.

3. Fishburn, P.C. (1977). "Condorcet Social Choice Functions", SIAM Journal of Applied Mathematics 33, 469-489.

4. Laffond, G., J.F. Laslier and M. Le Breton (1993). "The Bipartisan Set of a Tournament Game", Games and Economic Behavior 5, 182-201.

5. Laffond, G., J.F. Laslier and M. Le Breton (1995). "Condorcet Choice Correspondences: A Set-theoretical Comparison", Mathematical Social Sciences 30, 23-35.

6. Miller, N.R. (1980). "A New Solution Set for Tournaments and Majority Voting: Further Graph-Theoretical Approaches to the Theory of Voting", American Journal of Political Sciences 24, 68-96.

7. Owen, G. (1982). Game Theory, $2^{\text {nd }}$ ed. Academic Press, New York.

8. Peris J.E. and B. Subiza (1994). "Maximal Elements of not Necessarily Acyclic Binary Relations", Economics Letters 44, 385-388.

9. Peris, J.E. and B. Subiza (1999). "Condorcet choice Correspondences for Weak Tournaments", Social Choice and Welfare 16, 217-231. 
10. Schwartz, T. (1972). "Rationality and the Myth of the Maximum", Noûs 6, 97-117.

11. Schwartz, T. (1986). The Logic of Collective Choice, Columbia University Press, New York. 